\title{
The effect of roughness model on scattering properties of ice crystals
}

\author{
Igor Geogdzhayev*, Bastiaan van Diedenhoven \\ Columbia University, 2880 Broadway, New York, NY 10025, USA
}

\section{A R T I C L E I N F O}

Article history:

Received 28 September 2015

Received in revised form

1 March 2016

Accepted 1 March 2016

\begin{abstract}
A B S T R A C T
We compare stochastic models of microscale surface roughness assuming uniform and Weibull distributions of crystal facet tilt angles to calculate scattering by roughened hexagonal ice crystals using the geometric optics (GO) approximation. Both distributions are determined by similar roughness parameters, while the Weibull model depends on the additional shape parameter. Calculations were performed for two visible wavelengths ( $864 \mathrm{~nm}$ and $410 \mathrm{~nm}$ ) for roughness values between 0.2 and 0.7 and Weibull shape parameters between 0 and 1.0 for crystals with aspect ratios of $0.21,1$ and 4.8 . For this range of parameters we find that, for a given roughness level, varying the Weibull shape parameter can change the asymmetry parameter by up to about 0.05 . The largest effect of the shape parameter variation on the phase function is found in the backscattering region, while the degree of linear polarization is most affected at the side-scattering angles. For high roughness, scattering properties calculated using the uniform and Weibull models are in relatively close agreement for a given roughness parameter, especially when a Weibull shape parameter of 0.75 is used. For smaller roughness values, a shape parameter close to unity provides a better agreement. Notable differences are observed in the phase function over the scattering angle range from $5^{\circ}$ to $20^{\circ}$, where the uniform roughness model produces a plateau while the Weibull model does not.
\end{abstract}

(c) 2016 Elsevier Ltd. All rights reserved.

\section{Introduction}

Ice cloud feedbacks play an important role in global climate and are among the more important sources of uncertainty for modeling climate change [1-5]. Improving our understanding of the optical properties of ice clouds is thus an important component in the efforts to reduce this uncertainty through both climate modeling and remote sensing of clouds. Studies indicate that microscale surface roughness significantly affects the optical properties of ice crystals, smoothing out the scattering features, suppressing the formation of halos, and reducing the asymmetry parameters [6-10]. Remote sensing results indicate that the degree of surface roughness is generally high in tops of

\footnotetext{
* Corresponding author
}

natural ice clouds, but depends on location, temperature and atmospheric state [11,12]. Furthermore, laboratory studies [13-15] demonstrate that the microscopic structure of ice crystals is complex and highly dependent on the environmental conditions.

To calculate optical properties of crystals with arbitrary surfaces, numerically exact methods are available such as the discrete dipole approximation [16,18], the pseudospectral time domain method [17] and the invariant imbedding $T$-matrix method [19], but their application to particles with larger size parameters (defined as $\pi D / \lambda$, where $D$ is a characteristic length of the particle and $\lambda$ is the wavelength of light) is presently limited because of their computational burden. Approximate methods based on geometric optics (GO) $[20,21]$ are often used for larger particles. To account for crystal surface roughness, stochastic approaches are implemented in such GO 
applications. Liu et al. [22] demonstrated that such stochastic models are an efficient and relatively accurate means for simulating scattering properties of crystals with roughened surfaces. However, while such stochastic approaches have been implemented in available GO codes in significantly different ways $[20,21,23]$, a thorough investigation of the impact of the choice of roughness model on the scattering properties of ice crystals is not available to date. The goal of this study is to investigate how the choice of microscale roughness model affects calculated scattering properties of ice crystals.

In Section 2, we will first summarize different roughness models and discuss their interpretation before showing results in Section 3. We conclude the paper in Section 4.

\section{Approach}

To calculate scattering properties of hexagonal ice crystals we use the Monte Carlo ray-tracing code developed by Macke et al. [20,24]. In this study we used two models of surface roughness that are both based on randomly tilting the normal to the crystal surface by a certain angle at each ray reflection or refraction event. The two models differ in the assumed distribution of the random tilt angles. The model originally used in [20] assumes that the zenith tilt angle is distributed uniformly between $0^{\circ}$ and some maximum angle smaller than $90^{\circ}$ (i.e. $\sigma_{\text {uni }} \times 90^{\circ}$ ), where the parameter $\sigma_{\text {uni }}$ is commonly referred to as the roughness parameter. Shcherbakov et al. [23] performed calculations and an analysis of the scattering properties of ice crystals using the ray-tracing code from $[20,24]$ with a model of surface roughness implemented that is based on the twoparameter Weibull statistics [25]. In this model the cosine of the zenith tilt angle $\mu$ is generated using the following expression:

$\left.\mu=1 /\left[1+\sigma^{2}(-\ln t)^{1 / \eta}\right)\right]^{1 / 2}$,

where $t$ is a random number uniformly distributed on the interval $[0,1]$ and $\sigma$ and $\eta$ are two parameters determining the height and shape of the distribution, respectively.

In both the uniform and Weibull models the azimuth tilt angles are distributed uniformly between $[0,2 \pi]$. Parameter $\sigma_{\text {uni }}$ of the uniform distribution and parameter $\sigma$ of the Weibull distribution play similar roles and are referred to as the "roughness parameter" below. Parameter $\eta$ of the Weibull model modifies the shape of the distribution. In particular, setting $\eta=1$ results in a Gaussian distribution as used by, e.g. [21]. Neshyba et al. [13] demonstrated how the above parameters relate to a general mean surface normal roughness metric. The code does not handle the shadowing and ray re-entry effects associated with highly tilted facets that occur more frequently for high roughness parameters. For this reason we limit our calculations to roughness parameters smaller or equal than 0.7 .

Fig. 1 compares the relative frequency of occurrence of tilt angles as a function of the tilt angle in degrees for the two models with the roughness parameter of 0.2 (upper
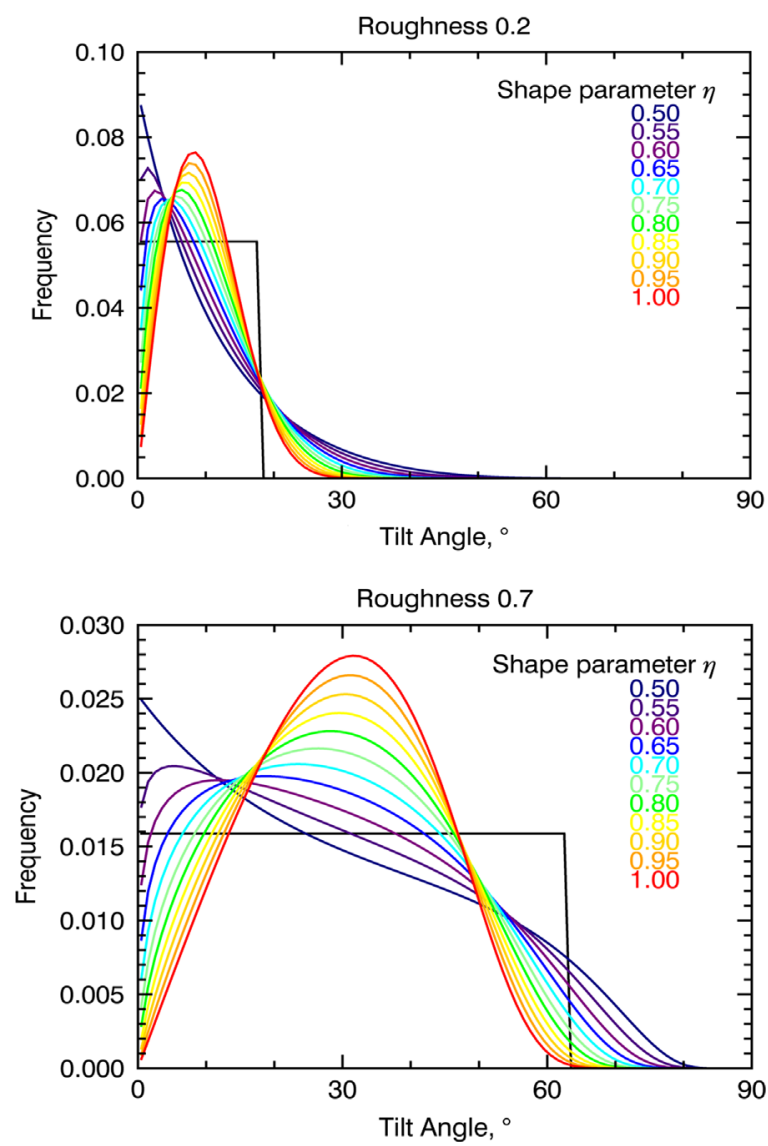

Fig. 1. Relative frequency of occurrence of tilts for the uniform (black line) and Weibull (colored lines) models of surface roughness for roughness parameter 0.2 (upper panel) and 0.7 (lower panel) and shape parameter $\eta$ varying between 0.5 and 1 . Note the difference in vertical scales. (For interpretation of the references to color in this figure legend, the reader is referred to the web version of this article.)

panel) and 0.7 (lower panel) and Weibull shape parameter $\eta$ between 0.5 and 1 . One can see that the uniform model is characterized by a sharp drop-off at the maximum tilt angle $\sigma_{\text {uni }} \times 90^{\circ}\left(18^{\circ}\right.$ and $63^{\circ}$, respectively) while the Weibull models result in a smooth distribution that covers all of the theoretically possible tilt angles. Furthermore, Weibull distributions with all but the smallest shape parameters result in a maximum in the tilt angles that is sharper than the uniform distribution with the same roughness. For the smaller roughness parameter, Weibull distributions show a significant proportion of tilts higher than the uniform threshold, while for the large roughness this is only true for $\eta$ smaller than approximately 0.75 .

It is of interest how the model roughness parameter relates to physical microscopically rough structures on the ice crystals. Within the framework of the GO approximation, the problem of determining such structures is reduced to constructing a physical crystal surface that, when illuminated by light rays, will result in a distribution of tilts equivalent to the one represented by the roughness model. The exact solution to this problem may be complex or may not exist because the shape of such a surface may potentially depend on the direction of incident and refracted rays. 
However, a simplified model of a physical surface may serve as a useful visualization of the differences between the theoretical models of roughness used in this study and elsewhere. We consider a surface completely covered by approximately hemispherical micro bulges. We then require that light rays vertically incident to the surface plane and spatially uniform would have angles of incidence on the bulges equivalent to the distribution of slopes in the theoretical model. Fig. 2 shows the equivalent surface shapes in arbitrary units for the model distributions presented in Fig. 1. Presented this way, the surfaces representing the uniform and Weibull distributions are strikingly similar, which is consistent with the findings of Neshyba et al. [13]. For both models the height of the bulges increases for higher roughness (note the difference in the vertical scale for the two panels). One can see, however, that for larger shape parameters $\eta$ the Weibull model results in bulges falling off from the maximum slower than the uniform model, while the opposite is true for smaller values of $\eta$. For larger roughness, extreme slopes are generally less likely for the Weibull distribution compared to the uniform one, resulting in gentler decreases at the sides of the bulges seen in Fig. 2, while the opposite is true for smaller roughness parameters.
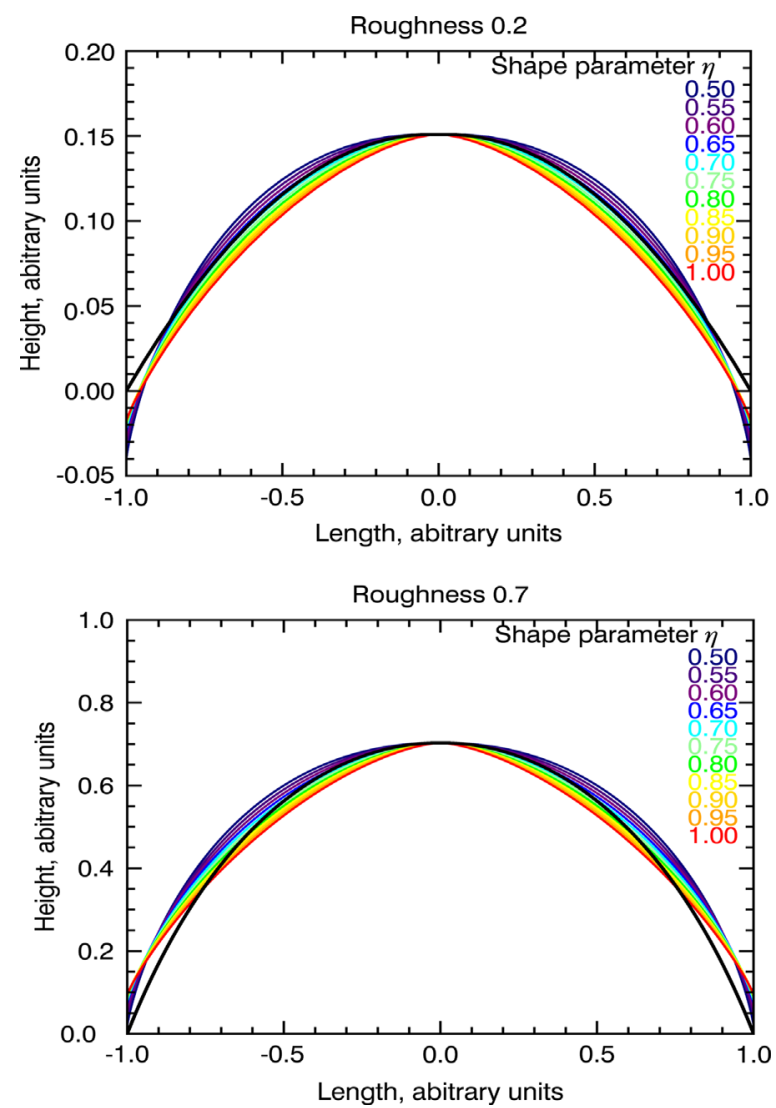

Fig. 2. Equivalent micro-surface shape for the uniform (black line) and Weibull (colored lines) models of surface roughness for roughness parameter 0.2 (upper panel) and 0.7 (lower panel) and shape parameter $\eta$ varying between 0.5 and 1 . Note the difference in vertical scales. (For interpretation of the references to color in this figure legend, the reader is referred to the web version of this article.)
To investigate the influence of the roughness model on the calculated ice crystal scattering properties, in this study we use our database of phase matrix, extinction, scattering and absorption coefficients of ice crystals at $865 \mathrm{~nm}$ and $410 \mathrm{~nm}$, initially created for the analysis of measurements of the Research Scanning Polarimeter (RSP) $[26,27]$. The calculations are performed using the geometric optics (GO) code [20] for randomly oriented hexagonal plates and columns with crystal sizes corresponding to equivalent-area spheres with radii between 5 and $320 \mu \mathrm{m}$. Note that the ray tracing part of calculations is independent of size for the wavelength considered here at which ice is essentially non-absorbing, while the diffraction at the projected cross-section included in the overall results does depend on the crystal size. Each calculation used 20,000 orientations and 32,000 rays per orientation. The aspect ratios of columns and plates (defined as the ratio of the height of the hexagonal prism to the diameter of the circle circumscribed around the hexagonal face of the prism) vary between 0.02 and 50 with 26 geometrically increasing steps for a total of 51 aspect ratios. The calculations are made for the uniform roughness model with the roughness parameter $\sigma_{\text {uni }}$ varying between 0 and 0.7 in steps of 0.05 . This results in around 10,000 calculations per wavelength. Calculations are also made for the entire parameter set as described above but using the Weibull model with the roughness parameter $\sigma$ varying between 0 and 0.7 in steps of 0.05 and the shape parameter $\eta=0.75$. This value of the shape parameter is chosen because it was reported in [23] to provide the best agreement with observational data. In addition, to investigate the influence of the Weibull shape parameter, we include calculations for $\eta$ varying between 0.5 and 1 at roughness values between 0.2 and 0.7 for hexagonal plates and columns with projected areas equivalent to a sphere with radius of $40 \mu \mathrm{m}$ and with aspect ratios of 0.21 and 4.8, respectively, as well as for a prism with unity aspect ratio.

\section{Results}

Figs. 3 and 4 show typical angular dependencies of the phase function and degree of linear polarization (DoLP), respectively, for the Weibull model with different shape parameters $\eta$ and roughness values of 0.2 and 0.7 and for the corresponding uniform roughness model. One can see that for the smaller roughness value significant differences exist between the uniform and the Weibull phase functions. At this roughness values of the $22^{\circ}$ and $46^{\circ}$ halo features are strongly suppressed for the uniform model [9] while they are still visible for Weibull model for all but the highest shape parameters. The two models also differ in the $120-140^{\circ}$ and the backscattering regions, where the uniform phase function is lower than the Weibull ones for any $\eta$. For the high roughness (lower panel) the halo features are absent for both models and the phase function calculated with the uniform roughness model is within the range of variation of the phase functions resulting from the Weibull model shape parameters from 0.5 to 1 . For this roughness value, the largest variations resulting from 

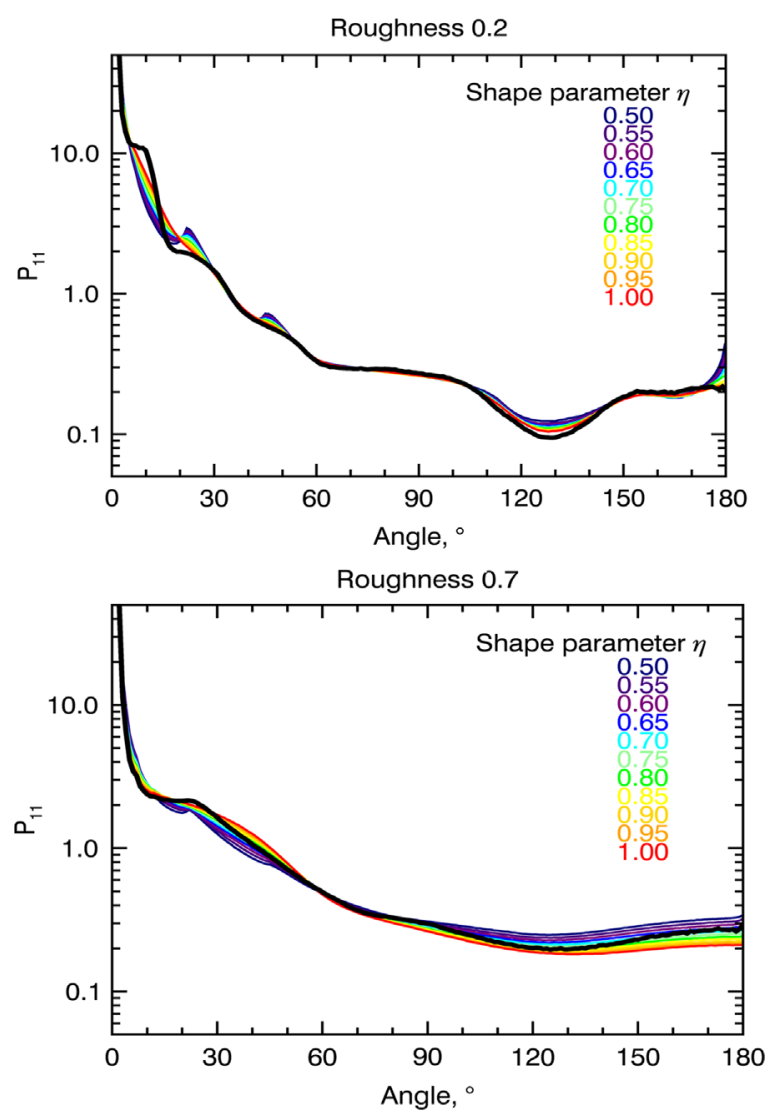

Fig. 3. Angular dependence of the phase function for the Weibull model with different shape parameters $\eta[0.5,1]$ (colored lines) and roughness 0.2 (upper panel) and 0.7 (lower panel) and for the corresponding uniform model (black lines). Hexagonal prism with unity aspect ratio corresponding to equivalent-area sphere with radius $40 \mu \mathrm{m}$. (For interpretation of the references to color in this figure legend, the reader is referred to the web version of this article.)

changing the Weibull model shape parameters occur in the back-scattering region at the scattering angles greater than $100^{\circ}$ as well as in the region between $30^{\circ}$ and $40^{\circ}$. Interestingly, the uniform model produces a plateau around $10-20^{\circ}$, while this feature is absent for the Weibull model phase functions. We hypothesize that this plateau is the result of the abrupt change in the uniform distribution at the maximum tilt angle $\sigma_{\text {uni }} \times 90^{\circ}$ (see Fig. 1 ). Furthermore, the phase function calculated with the uniform roughness model features a slight bump at scattering angles around $90^{\circ}$ that is not seen in the Weibull model phase functions.

Similarly to the phase function behavior, Fig. 4 shows that the angular dependence of DoLP for the uniform roughness model is largely similar to the DoLP variation for Weibull roughness models for the same values of the roughness parameter. In general, DoLP is significantly larger for the low roughness value of 0.2 . For this value, most variability due to the change in the shape parameter occurs for scattering angles between approximately $125^{\circ}$ and $145^{\circ}$. Yang and $\mathrm{Fu}[28]$ have shown that the DoLP of compact, pristine hexagonal prisms at these scattering angles are mostly determined by rays that undergo one,
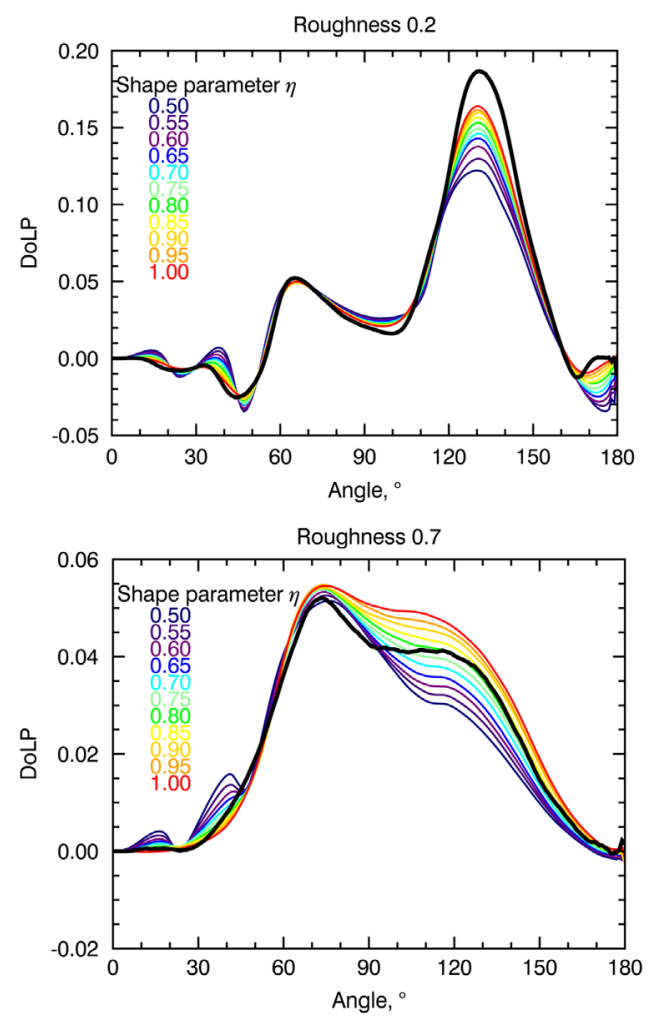

Fig. 4. Angular dependence of the DoLP for the Weibull model with different shape parameters $\eta[0.5,1]$ (colored lines) and roughness 0.2 (upper panel) and 0.7 (lower panel) and for the corresponding uniform model (black lines). Hexagonal prism with unity aspect ratio corresponding to equivalent-area sphere with radius $40 \mu \mathrm{m}$. (For interpretation of the references to color in this figure legend, the reader is referred to the web version of this article.)

two or three internal reflections. In this region the uniform model DoLP exceeds the Weibull model one for all considered shape parameters. For high roughness of 0.7 , the largest DoLP variation occurs at a wider range of sidescattering angles between approximately $80^{\circ}$ and $150^{\circ}$ and can be quite significant with differences in DoLP of between 0.03 and 0.045 at $120^{\circ}$. For high roughness, the DoLP resulting from the uniform roughness model features a dip around $90^{\circ}$ scattering angle, which is generally not or only weakly present in the Weibull results and corresponds to the bump seen in the uniform model phase function at these scattering angles (Fig. 3). The variation of DoLP with respect to roughness parameter using the uniform roughness model is also shown by [26], in addition to its variation with respect to aspect ratio.

It is of interest to investigate the joint effect of roughness and shape parameter changes on the optical properties of ice crystals. Fig. 5 shows the ratio of the asymmetry parameter for the Weibull model to the asymmetry parameter for the uniform model as a function of the shape parameter $\eta$ and roughness. Left panels are for the wavelength of $410 \mathrm{~nm}$, right panels are for $864 \mathrm{~nm}$. Top panels are for plates with the aspect ratio of 0.21 , middle panels are for crystals with aspect ratio of 1 , and bottom panels are for columns with the aspect ratio of 4.8. Overall, differences in asymmetry parameter of about $10 \%$ occur 

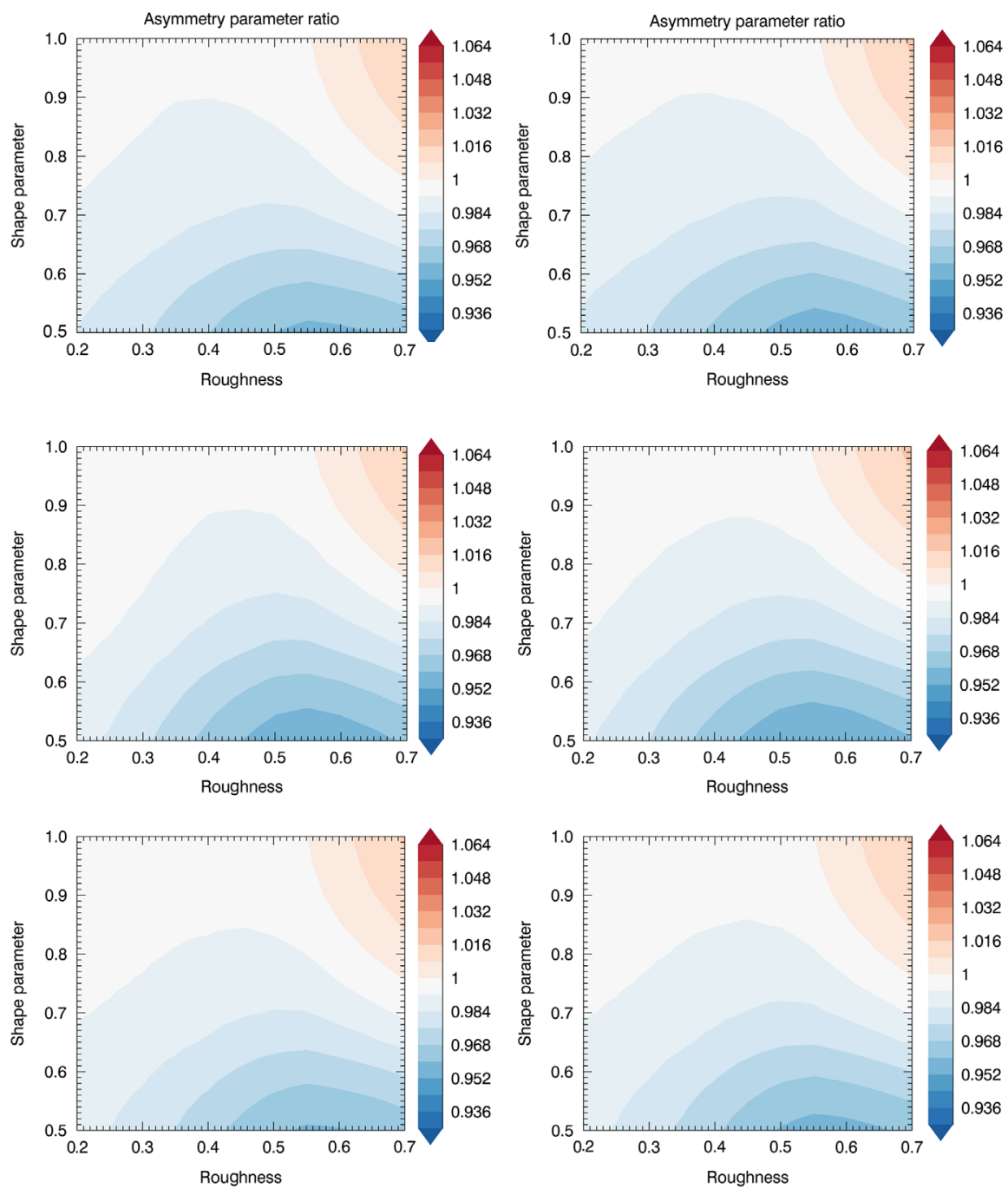

Fig. 5. Contour plots of the ratio of the asymmetry parameter for the Weibull model to the asymmetry parameter for the uniform model as a function of the shape parameter $\eta$ and roughness. Left panels are the wavelength of $410 \mathrm{~nm}$, right panels are for $864 \mathrm{~nm}$. Top panels are for plates with the aspect ratio of 4.8, middle panels are for crystals with aspect ratio of 1 , bottom panels are for columns with the aspect ratio of 4.8 .

between the two models and the patterns are remarkably similar for the two wavelengths and different aspect ratios. As one may expect, the effect of the shape parameter variation is small for less roughened crystals and increases for higher roughness parameters. For roughness parameters less than approximately 0.55 , the Weibull model results in generally smaller asymmetry parameters compared to the uniform distribution, while they exceed the corresponding values for the uniform model for larger roughness parameter and shape parameters larger than about 0.75 . For large roughness parameters, the two models result in similar asymmetry parameters when the shape parameter $\eta$ is around $0.7-0.85$. However, the shape parameter that provides the smallest differences in asymmetry parameter between the Weibull and uniform models depends on the roughness parameter. In order to further compare the two roughness models in the following, we use a shape parameter of 0.75 , since for that value the Weibull model produces asymmetry parameters comparable to the uniform model for both low and high roughness parameters.

Fig. 6 shows a contour plot of the asymmetry parameter integrated over size for the uniform model (upper panel), Weibull model with $\eta=0.75$ (middle panel) for various values of roughness and aspect ratio, as well as their difference (lower panel). One can see that the two models yield very similar ranges of asymmetry parameter as well as similar dependencies on aspect ratio, with a mean absolute difference of 0.006 (lower Panel). For this choice of shape parameter, the Weibull asymmetry parameter is slightly below the value for the uniform distribution for the same roughness, except for very small and very large roughness parameters, as was already concluded from Fig. 5. Some substantial maximum differences of up to about 0.015 are obtained around roughness values of 0.5 and in particular for plates with aspect ratio of approximately 0.4 . Results for both models exhibit a valley pattern aligned along the roughness parameter axis with 

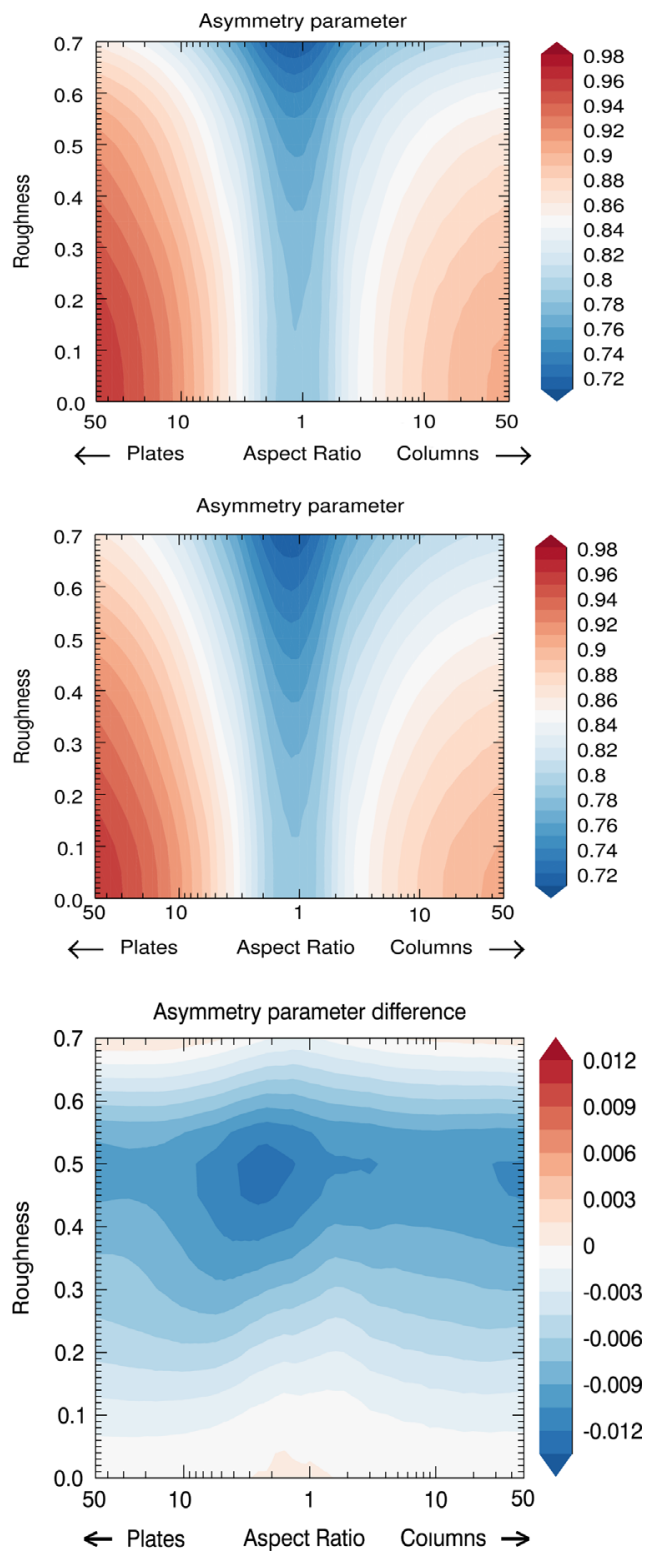

Fig. 6. The asymmetry parameter for the uniform (upper panel) and Weibull with $\eta=0.75$ (middle panel) roughness model for various values of roughness and aspect ratios. Also shown is the Weibull minus uniform asymmetry parameter differences (lower panel). Reciprocal aspect ratio is given for plates.

smaller asymmetry parameters corresponding to crystals with aspect ratios closer to unity and with increased roughness. The increase of asymmetry parameter with aspect ratio deviating from unity is somewhat stronger for plates than for columns when plotted on a logarithmic scale as in Fig. 6, which is in agreement with the results in $[20,28]$. This is true for both roughness models and is due to the larger parallel surfaces of plates resulting in a greater probability of light passing through the particle with a minimal change of direction [28].
The results of Figs. 5 and 6 indicate that the Weibull model with shape parameter $\eta$ of approximately 0.75 provides relatively close agreement with the uniform roughness model for highly roughened crystals of various aspect ratios in visible light. For smaller roughness, $\eta$ values close to unity provide the best agreement. It is of interest to put this observation in the context of previous studies. Baran and Labonnote [29] found that global satellite-measured polarized reflectances of ice clouds are best modeled using $\eta=0.85$ and $\sigma=0.4$ using a crystal ensemble model with inclusions. Neshyba et al. [13] found that $\eta$ between 0.75 and 1 with $\sigma=0.4-0.5$ is needed to match electron microscope observation of ice crystals roughness. Using nephelometer measurements of scattering by ice crystal, Shcherbakov et al. [23] retrieved Weibull shape parameter values of between 0.73 and 0.77 with $\sigma=0.05-0.25$. Thus, available measurements are generally consistent with shape parameter values of about 0.7-0.85.

Fig. 7 shows the surface plot of the angular dependence of the size-integrated phase function for columns and plates with different aspect ratios and a roughness parameter of 0.2. The forward peak is truncated. The upper panel corresponds to the uniform roughness model, while the lower panel is for the Weibull model with shape parameter 0.75 . The figure also shows the degree of linear polarization color-coded according to the color bar on the right. One important difference between the panels of Fig. 7 is the presence of a plateau at scattering angles between $5^{\circ}$ and $20^{\circ}$ for the uniform tilt model, while no such feature is visible for the Weibull model. This plateau is also observed in Fig. 3 for crystals of unity aspect ratio and appears to be a general artifact of the uniform roughness model for all aspect ratios. Other differences include somewhat higher DoLP for the uniform model for crystals with extreme aspect ratios, especially for columns. Furthermore, a slight increase in DoLP towards backscattering visible for the uniform model is absent for the Weibull model.

\section{Conclusions}

We investigated how the choice of roughness model affects scattering properties of hexagonal ice crystals using the geometric optics approximation. We compared the influence on the phase function and DoLP by surface roughness based on a one-parameter uniform distribution and a two-parameter Weibull distribution of random surface tilts. Note that the commonly used Gaussian distribution of crystal facets tilts is a special case of the Weibull distribution. Calculations were performed at $865 \mathrm{~nm}$ and $410 \mathrm{~nm}$ for a representative set of ice crystal sizes and aspect ratios. We found that for strongly roughened crystals varying the Weibull shape parameter between 0.5 and 1.0 results in a $7 \%$ change in the asymmetry parameter compared to that calculated using the uniform model value. For this range the largest variations in the phase functions are found in the backscattering region, while the DoLP is most variable at the side- 

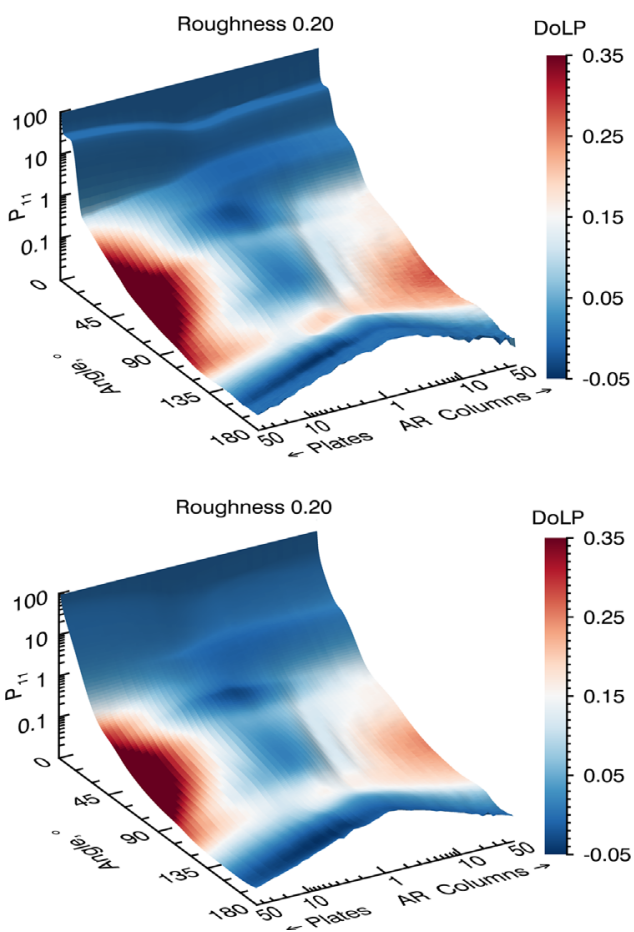

Fig. 7. Phase function (surface) and DoLP (color) for the uniform (upper panel) and Weibull (lower panel) tilt models vs aspect ratio for roughness 0.2. Reciprocal of aspect ratio is given for plates. (For interpretation of the references to color in this figure legend, the reader is referred to the web version of this article.)

scattering angles. For high roughness parameter of about $0.55-0.7$, a value of around 0.75 for the shape parameter of the Weibull distribution produces the closest overall agreement in asymmetry parameter, phase function and DoLP between the two models of roughness. For smaller roughness shape parameters close to 1 provide a better match between the two models. However, when roughness is small significant differences in the phase function and DoLP at side-scattering angles exist between the uniform and Weibull models for any of the considered shape parameters. Notable differences are observed in the angular dependence of the phase function at $5-20^{\circ}$ scattering angles for crystals of all aspect ratios, where the uniform tilt model produces a plateau while the Weibull model does not.

The results presented here are based on single hexagonal prisms with various aspect ratios, while more complex crystals are generally found in observed ice clouds. However, it has been shown previously that complex ice crystals that consist of hexagonal components have scattering phase matrices that closely resemble the scattering phase matrices of the individual components [3,30-32]. Thus, the conclusions presented here are likely qualitatively applicable to more complex structures such as aggregates of columns, aggregates of plates and bullet rosettes.

Compared to the influence that the level of roughness and the crystal aspect ratio have on phase functions and the DoLP, the effects due to choice of roughness model are relatively small. This suggests that the choice of roughness model would have a relatively minor effect on, e.g., satellite retrievals of ice cloud properties and calculations of ice cloud radiative effects. However, subtle differences in the DoLP functions between roughness models may influence inferences of crystal geometry, roughness parameters and asymmetry parameters from polarized reflectances measured by airborne or satellite-based polarimeters $[11,12,26,27]$. Furthermore, the presented differences in phase functions in the forward direction may influence interpretations of ground-based measurements [33,34].

\section{Acknowledgments}

We would like to acknowledge support of the work presented here under pre-formulation funding of the Aerosol Cloud Ecosystem (ACE) mission by NASA Headquarters and the ACE program scientists Hal Maring and Paula Bontempi. This material is also based upon work supported by the NASA ROSES program element A28 (The science of Aqua and Terra) under Grant number NNX14AJ28G. We thank Valery Shcherbakov for providing code on which we based the implementation of the Weibull model in our GO code. We thank two anonymous reviewers for their insightful comments that have resulted in a much-improved manuscript.

\section{References}

[1] Stephens GL, Tsay S-C, Stackhouse Jr PW, Flatau PJ. The relevance of the microphysical and radiative properties of cirrus clouds to climate and climatic feedback. J Atmos Sci 1990;47:1742-54.

[2] Vogelmann AM, Ackerman TP. Relating cirrus cloud properties to observed fluxes: a critical assessment. J Atmos Sci 1995;52:4285-301.

[3] Fu Q. A new parameterization of an asymmetry factor of cirrus clouds for climate models. J Atmos Sci 2007;64:4140. http://dx.doi.org/10. 1175/2007JAS2289.1.

[4] Waliser DE, Li J-LF, Woods CP, Austin RT, Bacmeister J, Chern J, Del Genio A, Jiang JH, Kuang Z, Meng H, Minnis P, Platnick S, Rossow WB, Stephens GL, Sun- Mack S, Tao W-K, Tompkins AM, Vane DG, Walker C, Wu D. Cloud ice: a climate model challenge with signs and expectations of progress. J Geophys Res 2009;114:D00A21. http://dx.doi.org/ 10.1029/2008JD010015.

[5] Baran AJ. From the single-scattering properties of ice crystals to climate prediction: a way forward. Atmos Res 2012;112:45-69.

[6] Baum BA, Yang P, Hu Y-X, Feng Q. The impact of ice particle roughness on the scattering phase matrix. J Quant Spectrosc Radiat Transf 2010;111:2534-49.

[7] Yi BQ, Yang P, Baum BA, L'Ecuyer T, Oreopoulos L, Mlawer EJ, et al. Influence of ice particle surface roughening on the global cloud radiative effect. J Atmos Sci 2013;70:2794-807.

[8] Shcherbakov V. Why the $46^{\circ}$ halo is seen far less often than the $22^{\circ}$ halo? J Quant Spectrosc Radiat Transf 2013;124: 37-44. http://dx.doi.org/10.1016/j.jqsrt.2013.03.002.

[9] Van Diedenhoven B. The prevalence of the $22^{\circ}$ halo in cirrus clouds. J Quant Spectrosc Radiat Transf 2014;146:475-9. http://dx.doi.org/ 10.1016/j.jqsrt.2014.01.012.

[10] Van Diedenhoven B, Ackerman AS, Cairns B, Fridlind AM. A flexible parameterization for shortwave optical properties of ice crystals. J Atmos Sci 2014;71(5):1763-82. http://dx.doi.org/10.1175/JAS-D-130205.1.

[11] Cole BH, Yang P, Baum BA, Riedi J, Labonnote LC. Ice particle habit and surface roughness derived from PARASOL polarization measurements. Atm Chem Phys 2014;14(7):3739-50. http://dx.doi.org/10.5194/acp14-3739-2014.

[12] Van Diedenhoven B, Fridlind AM, Cairns B, Ackerman AS. Variation of ice crystal size, shape and asymmetry parameter in tops of tropical deep convective clouds. J Geophys Res Atmos 2014;119(20): 11809-25. http://dx.doi.org/10.1002/2014JD022385. 
[13] Neshyba SP, Lowen B, Benning M, Lawson A, Rowe PM. Roughness metrics of prismatic facets of ice. J Geophys Res 2013;118: 3309-18. http://dx.doi.org/10.1002/jgrd.50357.

[14] Pfalzgraff WC, Hulscher RM, Neshyba SP. Scanning electron microscopy and molecular dynamics of surfaces of growing and ablating hexagonal ice crystals. Atmos Chem Phys 2010;10:2927-35.

[15] Magee NB, Miller A, Amaral M, Cumiskey A. Mesoscopic surface roughness of ice crystals pervasive across a wide range of ice crystal conditions. Atmos Chem Phys 2014;14:12357-71.

[16] Yurkin MA, Hoekstra AG, Scott Brock R, Lu JQ. Systematic comparison of the discrete dipole approximation and the finite difference time domain method for large dielectric scatterers. Opt Express 2007;15(17):902-11.

[17] Liu C, Bi L, Panetta RL, Yang P, Yurkin MA. Comparison between the pseudo-spectral time domain method and the discrete dipole approximation for light scattering simulations. Opt Express 2012;20 (15):16763-76.

[18] Bi L, Yang P. Accurate simulation of the optical properties of atmospheric ice crystals with the invariant imbedding T-matrix method. J Quant Spectrosc Radiat Transf 2014;138:17-35.

[19] Um J, McFarquhar GM. Formation of atmospheric halos and applicability of geometric optics for calculating single-scattering properties of hexagonal ice crystals: Impacts of aspect ratio and ice crystal size. J Quant Spectrosc Radiat Transf 2015;165:134-52.

[20] Macke A, Mueller J, Raschke E. Single scattering properties of atmospheric ice crystals. J Atmos Sci 1996;53:2813-25.

[21] Yang P, Liou KN. Geometric-optics-integral-equation method for light scattering by nonspherical ice crystals. Appl Opt 1996;35(33): 6568-84.

[22] Liu C, Panetta RL, Yang P. The effects of surface roughness on the scattering properties of hexagonal columns with sizes from the Rayleigh to the geometric optics regimes. J Quant Spectrosc Radiat Transf 2013;129:169-85.

[23] Shcherbakov V, Gayet J-F, Baker B, Lawson P. Light Scattering by Single Natural Ice Crystals. J Atmos Sci 2006;63:1513-25. http://dx.doi.org/ 10.1175/JAS3690.1.

[24] Macke A, Mishchenko MI, Cairns B. The influence of inclusions on light scattering by large ice particles. J Geophys Res 1996;101(23): 311-6.
[25] Hazewinkel M, editor. Encyclopaedia of mathematics. Dordrecht Boston/London: Kluwer Academic Publishers; 1993.

[26] Van Diedenhoven B, Cairns B, Geogdzhayev IV B, Fridlind AM, Ackerman AS, Yang P, Baum BA. Remote sensing of ice crystal asymmetry parameter using multi-directional polarization measurements. Part I: methodology and evaluation with simulated measurements. Atmos Meas Tech 2012;5:2361-74. http://dx.doi.org/10.5194/amt-5-23612012.

[27] Van Diedenhoven B, Cairns B, Fridlind AM, Ackerman AS, Garrett T] Remote sensing of ice crystal asymmetry parameter using multidirectional polarization measurements - Part 2: application to the research scanning polarimeter. Atmos Chem Phys 2013;13: 3185-203. http://dx.doi.org/10.5194/acp-13-3185-2013.

[28] Yang P, Fu Q. Dependence of ice crystal optical properties on particle aspect ratio. J Quant Spectrosc Radiat Transf 2009;110: 1604-14. http://dx.doi.org/10.1016/j.jqsrt.2009.03.004.

[29] Baran AJ, Labonnote L-C. A self-consistent scattering model for cirrus. I: The solar region. Q J R Meteorol Soc 2007;133(629): 1899-912. http://dx.doi.org/10.1002/qj.164.

[30] Iaquinta J, Isaka H, Personne P. Scattering phase function of bullet rosette ice crystals. J Atmos Sci 1995;52(9):1401-13.

[31] Um J, McFarquhar GM. Single-scattering properties of aggregates of plates. Q J R Meteorol Soc 2009;135(639):291-304. http://dx.doi.org/ $10.1002 /$ qj.378.

[32] Baran AJ. A review of the light scattering properties of cirrus. J Quant Spectrosc Radiat Transf 2009;110(14-16):1239-60. http://dx.doi.org/ 10.1016/j.jqsrt.2009.02.026.

[33] Segal-Rosenheimer M, Russell PB, Livingston JM, Ramachandran S, Redemann J, Baum BA. Retrieval of cirrus properties by Sun photometry: A new perspective on an old issue. J Geophys Res Atmos 2013;118:4503-20. http://dx.doi.org/10.1002/jgrd.50185.

[34] Knobelspiesse K, van Diedenhoven B, Marshak A, Dunagan S, Holben B, Slutsker I. Cloud thermodynamic phase detection with polarimetrically sensitive passive sky radiometers. Atmos Meas Tech 2015;8:1537-54. http://dx.doi.org/10.5194/amt-8-1537-2015. 